\title{
Cardiac nonamyloidotic immunoglobulin deposition disease
}

\author{
Amir A Toor ${ }^{1,6}$, Ben A Ramdane ${ }^{1}$, Jacob Joseph², Maria Thomas³ ${ }^{3}$ Carl O’Hara ${ }^{4}$, \\ Bart Barlogie $^{1}$, Patrick Walker ${ }^{5}$ and Lija Joseph ${ }^{3,4,7}$ \\ ${ }^{1}$ Myeloma Institute for Research and Therapy, University of Arkansas for Medical Sciences, Little Rock, \\ AR, USA; ${ }^{2}$ Division of Cardiovascular Medicine, Boston University School of Medicine, Boston, MA, USA; \\ ${ }^{3}$ Department of Pathology, University of Arkansas for Medical Sciences, Little Rock, AR, USA; ${ }^{4}$ Department \\ of Pathology, Mallory Institute of Pathology, Boston Medical Center, Boston University School of Medicine, \\ Boston, MA, USA and ${ }^{5}$ Nephropathology Associates, Little Rock, AR, USA
}

\begin{abstract}
Cardiac nonamyloidotic immunoglobulin (Ig) deposition disease (CIDD) is a rare disorder characterized by Ig deposition in the myocardium associated with plasma cell dyscrasias. A retrospective review of cardiac biopsies performed at two different institutions identified eight patients with CIDD. All patients had plasma cell dyscrasias with monoclonal gammopathy. Three had $\lg G \lambda$, two had $\lg G \kappa$, one had $\lg D \kappa$ and one each had free $\kappa$ and free $\lambda$ light chain. Four patients had concurrent amyloidosis involving other organs. One had amyloidosis of kidney alone, one had amyloidosis of kidney and abdominal fat pad and two others had amyloidosis of bone marrow vasculature. Three patients had dialysis-dependent renal insufficiency. None of the patients had symptoms of heart failure. Six patients had echocardiographically demonstrable concentric left ventricular hypertrophy with diastolic dysfunction. Two patients had significant cardiac arrhythmias requiring medical intervention. On endomyocardial biopsy, all eight had normal appearing myocardium on light microscopy with negative Congo Red and Thioflavin T stains. On immunofluorescent staining of the cardiac biopsies, all eight stained positive for interstitial Ig deposition. Electron microscopy (EM) confirmed the presence of granular deposits of Igs in the myocardium in five of the eight patients. EM studies were not available in one patient and two others had normal EM studies. In conclusion, CIDD should be considered in the spectrum of cardiovascular pathology in patients with plasma cell dyscrasias. They often, but not always, have left ventricular hypertrophy. These patients may be at risk for developing arrhythmias as well as diastolic dysfunction. Unless immunofluorescent and EM studies are performed routinely in biopsy material, this entity may be missed in the absence of amyloidosis. Concurrent amyloidosis in other organs sheds a unique perspective into the role of local microenvironment in the pathogenesis of systemic Ig deposition disease and amyloidosis.

Modern Pathology (2006) 19, 233-237. doi:10.1038/modpathol.3800524; published online 2 December 2005
\end{abstract}

Keywords: myocardium; immunoglobulin deposition disease; amyloid; light-chain deposition disease; arrhythmia; left ventricular hypertrophy

The secretion of abnormal monoclonal immunoglobulins (Igs) usually characterizes plasma cell dyscrasias. This can give rise to a variety of complications ranging from cast nephropathy to various forms of monoclonal Ig deposition dis-

Correspondence: Dr L Joseph, MD, Department of Pathology, Mallory Institute of Pathology, Boston Medical Center, Boston University School of Medicine, Room 330A, 784 Albany Street, Boston, MA 02118, USA.

E-mail: Lija.Joseph@bmc.org

${ }^{6}$ Current address: Department of Hematology and Oncology, Loyola University Stritch School of Medicine, Chicago, IL, USA

${ }^{7}$ Current address: Department of Pathology, Boston University School of Medicine, Boston, MA, USA

Received 25 May 2005; revised 28 September 2005; accepted 19

October 2005; published online 2 December 2005 eases. $^{1,2}$ Specifically, in the heart, Igs can be deposited as AL amyloid, light-chain deposition disease or light- and heavy-chain deposition disease. ${ }^{3}$ Congo Red and Thioflavin $\mathrm{T}$ positivity as well as nonbranching fibrillary structure of the protein deposition by electron microscopic (EM) evaluation characterizes amyloidosis. However, if the deposits are Congo Red and Thioflavin $\mathrm{T}$ negative, amorphous granular electron-dense deposits by EM and positive by immunofluorescence studies for light and/or heavy chains, it is designated cardiac nonamyloidotic Ig deposition disease (CIDD). Synonyms include light chain or light- and heavy-chain deposition disease. In this review, we restrict the term Ig deposition disease to the nonamyloidotic light chain or light- and heavy-chain deposition 
type. We report the clinical features, light, immunofluorescent and EM findings of eight patients who have CIDD diagnosed by myocardial biopsy. This is the largest series of such patients reported in literature to date to the best of our knowledge.

\section{Patients and methods}

For this study, we retrospectively collected the pathology reports of all the endomyocardial biopsies performed at our institutions. The biopsy results were then reviewed and all the patients referred for biopsy to rule out cardiac amyloidosis were identified. Of these patients, those with CIDD were then studied in detail by chart review.

Initial evaluation of all the patients included a detailed history and physical examination along with a comprehensive laboratory evaluation, which included complete blood counts, serum electrolytes, creatinine, 24-h creatinine clearance, serum and urine protein electrophoresis with immunofixation studies. All the patients had a bone marrow aspirate and biopsy, skeletal surveys and skull, spine and pelvis magnetic resonance imaging (MRI). Electrocardiogram and transthoracic echocardiography to evaluate cardiac function were also carried out. Patients with clinical symptoms or signs suggestive of amyloidosis underwent biopsy of the relevant organs on the discretion of the attending oncologist and consulting physicians (usually a cardiologist or a nephrologist). The indications for a biopsy included diastolic dysfunction, low ejection fraction, involvement of other areas by amyloid or for prognostication as documented in the patient chart.

The biopsy was processed for light, immunofluorescence and EM studies.

Endomyocardial biopsies for light microscopy were formalin fixed (10\% neutral-buffered formalin) and processed for hematoxylin and eosin (H\&E) staining. Congo Red and Thioflavin $\mathrm{T}$ stains to assess amyloid deposition were also performed. A portion of the biopsy was snap frozen in liquid nitrogen and immunofluorescent staining for IgG, IgA, IgM, $\kappa$ and $\lambda$ light chains were performed. EM studies were carried out on glutaraldehyde-fixed material. Amyloidosis was defined as the presence of fibrillar material in the cardiac interstitium or vascular walls that was Congo Red and Thioflavin $\mathrm{T}$ positive. CIDD was defined as the presence of immunofluorescent Ig deposition in the cardiac interstitium or vascular walls in the absence of amyloid as evaluated by Congo Red, Thioflavin $\mathrm{T}$ and EM examination. The EM showed granular electron-dense deposits.

\section{Results}

One of the institutions (University of Arkansas for Medical Sciences) evaluates about 300 new and referred myeloma patients each year. The other (Boston Medical Center) evaluates about 150 new and referred amyloid patients each year. Of the patients at UAMS, 30 underwent endomyocardial biopsies between August 1997 and June 2000. Of these 30 , six who were biopsied $(20 \%)$ had CIDD, seven $(31 \%)$ had cardiac amyloidosis and $17(49 \%)$ had neither disease identified. A new patient who was diagnosed since June 2000 was added to this study. One case was identified from Boston Medical Center pathology files.

Results are summarized in Table 1. The eight patients with Ig deposition disease had a median age of 49.5 years (range 40-64 years). There were five males and three females. All patients had plasma cell dyscrasias, five with diagnostic criteria of multiple myeloma, two meeting the diagnostic criteria for smoldering myeloma and one patient referred for amyloidosis. Three patients had $\operatorname{IgG} \lambda$, two with $\operatorname{IgG} \kappa$, and one each $\operatorname{IgD} \kappa, \kappa$ and $\lambda$ monoclonal protein.

None of the patients were symptomatic from their cardiac Ig deposition at presentation. However, on echocardiography, six patients had concentric left ventricular hypertrophy with diastolic dysfunction. Interventricular septal thickness was $15 \mathrm{~mm}$ in the four patients where it could be measured accurately (range 11-18). In two patients, the echocardiographic studies were reported as normal. Four patients had hypertension and four did not.

Endomyocardial biopsies of all eight patients had normal appearing myocardium on light microscopy H\&E stains (Figure 1). Congo Red (Figure 2) and Thioflavin $\mathrm{T}$ stains as well as EM examination were negative for amyloidosis. Immunofluorescent staining of the cardiac biopsies, IgG was present in four patients and IgD in one patient (Figure 3). Four were positive for $\lambda$ light chains and four for $\kappa$ light chains corresponding to the light chain found in the patient's serum. The deposits were seen primarily in the interstitium. Positive IgG heavychain staining was seen in four of the five patients with IgG heavy chain. On EM, five patients had granular electron-dense deposits in the interstitium (Figure 4), and two patients had normal myocardium by EM. One did not have EM studies available for review.

Interestingly, four patients had concurrent amyloidosis involving other organs. Four patients had renal biopsies to evaluate proteinuria. Of these, two had renal amyloidosis and two had light-chain deposition disease. Four patients had an abdominal fat pad aspirate, one of which showed Congo Redpositive amyloid deposits. Two patients had gastrointestinal involvement with amyloid. Two patients had amyloid deposition identified in their bone marrow.

One patient was noted to have had an episode of $15 \mathrm{~s}, 42$ beat run of spontaneous asymptomatic ventricular tachycardia when undergoing cardiac 


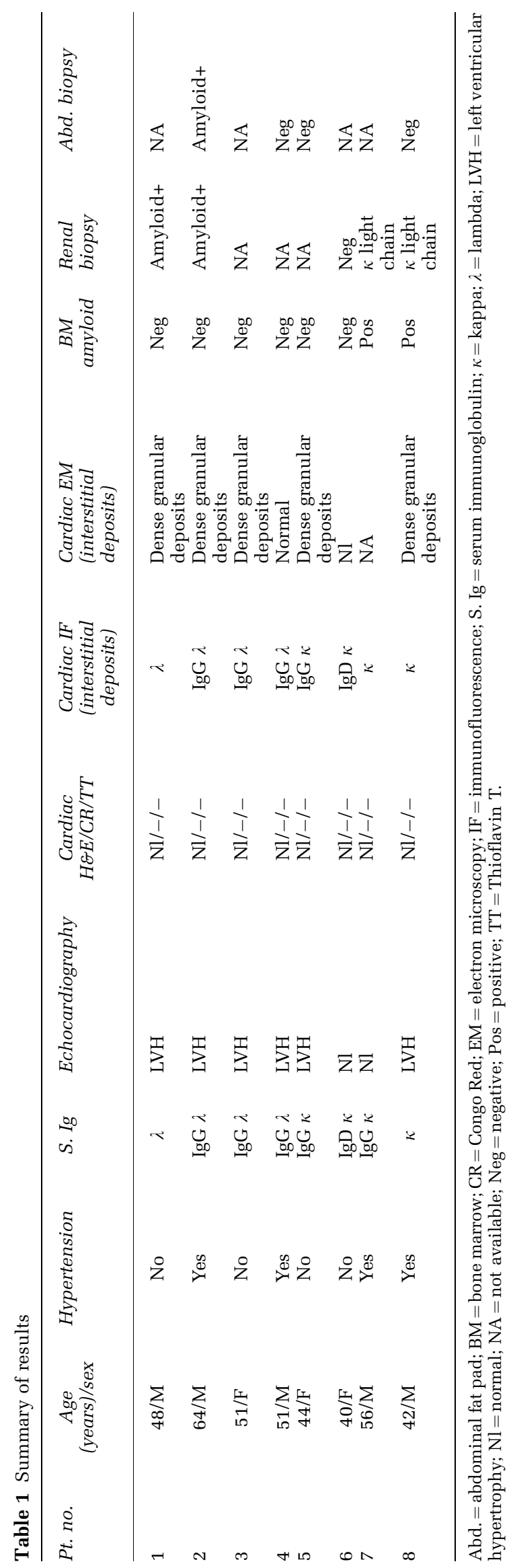

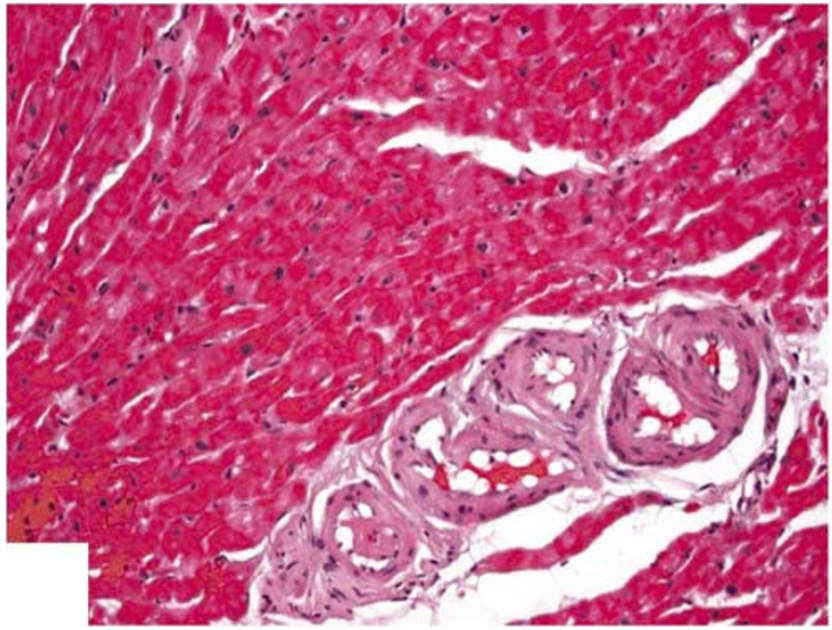

Figure $1 \mathrm{H} \&$ E-stained section of myocardium with normal myocytes and vasculature from a patient with CIDD. Interstitial deposits of $\kappa$ light chains were subsequently identified by immunoflourescence and EM $(\times 200)$.

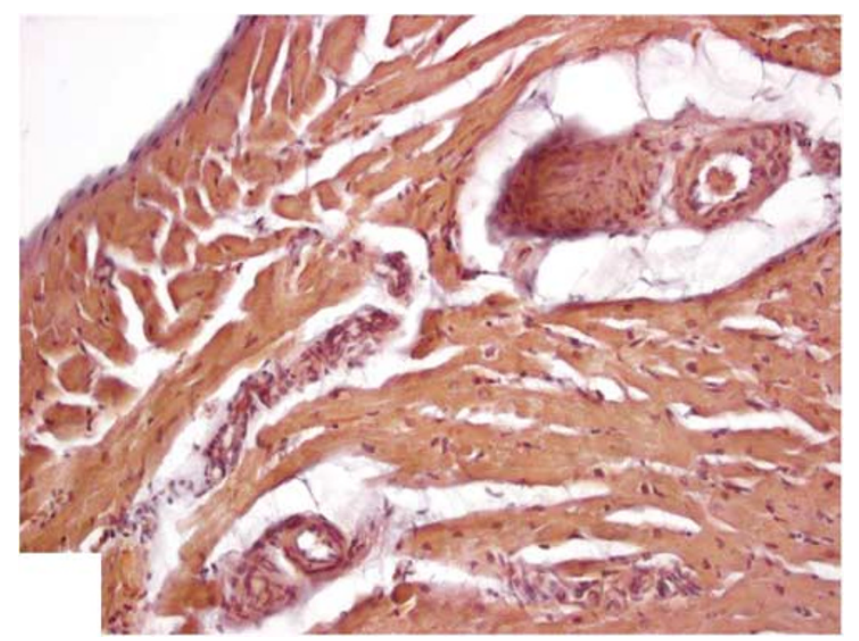

Figure 2 Congo Red-stained section of above patient from Figure 1 showing myocytes and vasculature that are Congo Red negative. There was no apple green birefringence with polarized light $(\times 200)$.

monitoring after admission for a presyncopal event, which occurred during chemotherapy administration (infusional cyclophosphamide, adriamycin, dexamethasone). Given his documented CIDD and this clinical event, an implanted cardioverter defibrillator device was placed. Another patient developed atrial fibrillation during chemotherapy (infusional cyclophosphamide, etoposide, dexamethasone and cisplatin). He responded to therapy with digoxin. Arrhythmogenesis during infusional chemotherapy in patients with CIDD has not been reported before. We do not know if this is truly related to each other or whether it is a random association at this time. 


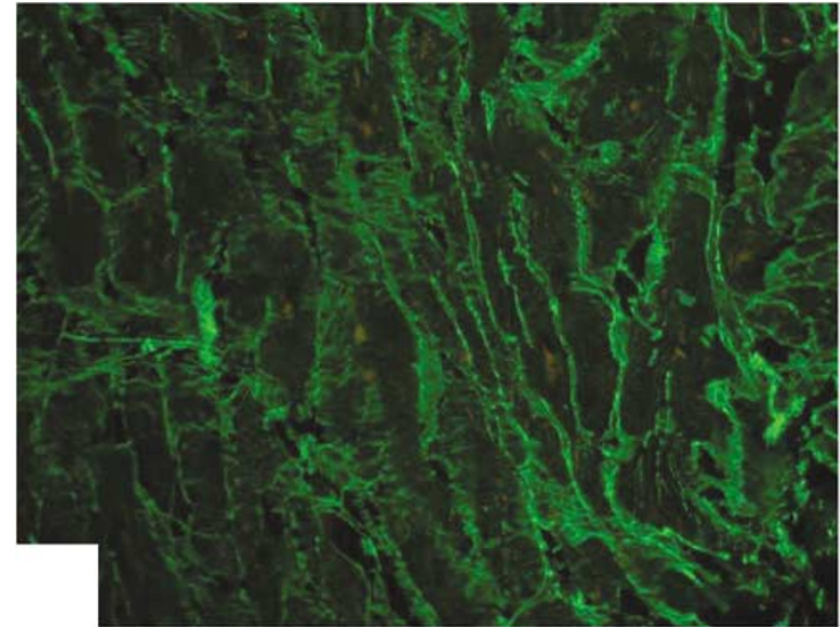

Figure 3 Immunofluorescence studies of the same patient from Figures 1 and 2 showing interstitial positivity for $\kappa$ light chain. Similar patterns were noted for heavy chains or $\lambda$ light chain in other patients $(\times 200)$.

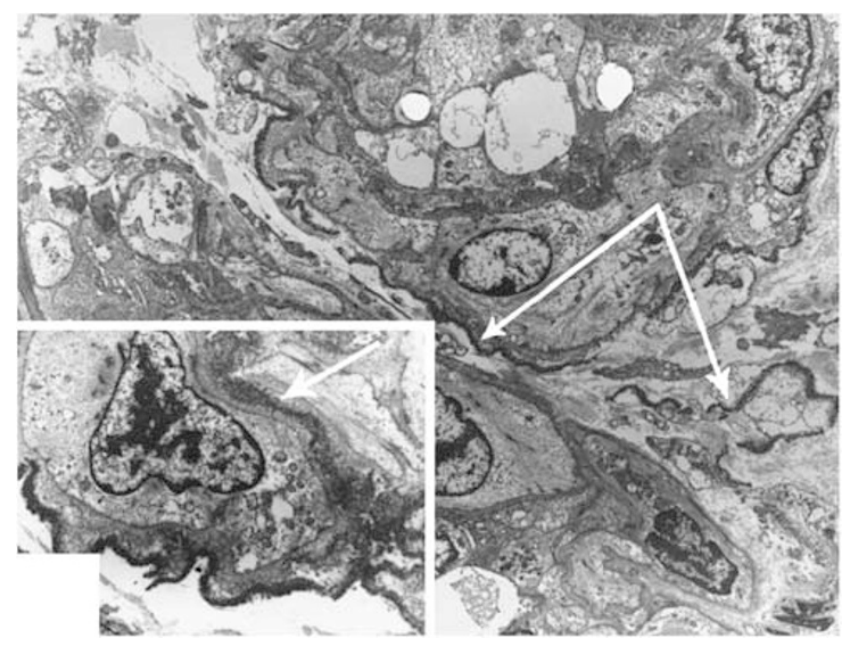

Figure 4 Low- and high-magnification electron micrographs showing extracellular granular deposits of Ig, with arrows pointing to the areas of granular electron-dense deposits $(\times 8800$; inset $\times 28200)$.

\section{Discussion}

Manifestations of Ig deposition disease in organs can be varied. It can present as a nephrotic syndrome, ${ }^{4}$ retinal detachment, ${ }^{5}$ cholestatic liver disease ${ }^{6}{ }^{\text {con- }}$ gestive cardiac failure, ${ }^{7,8}$ arrhythmias, ${ }^{9}$ neuropathy ${ }^{10}$ or Sicca syndrome ${ }^{11}$

One report of this deposition associated with marked systemic calcification is also noted in the literature. $^{12}$

Ig deposition in the myocardium is an uncommon but well described entity. ${ }^{1-3,7-9}$ The nonfibrillar Ig deposition may be composed of light chains, heavy chains or both. As is seen in our study, the Igs may be deposited as amyloid concurrently in other organs, specifically kidney, gastrointestinal tract, abdominal fat pad or bone marrow. The mechanisms by which in some patients the light chains assume a fibrillar configuration and hence form amyloid, and do not do so in others and also why they assume varied structure in different organs of the same patient are not completely clear at this time.

Gallo and Vidal et al ${ }^{13,14}$ did biochemical analysis of the nonfibrillar-type of $\kappa$ light chains deposited in the cardiac interstitium. They suggest that somatic mutations of specific amino-acid sequences in the light chains affect the folding and three-dimensional structure of the protein. This may determine the nature of light-chain tissue deposition. Based on in vivo and in vitro data, they postulate that Ig deposition disease may be an arrested form of preamyloid in the pathway of amyloid formation. However, the role of cardiac interstitium and cytokines in this interaction needs further study. In vitro studies in the kidneys by Herrera et al show that processing of the Ig by the mesangial cell and subsequent cellular signaling pathways play an important role in the distinction of the deposits. ${ }^{15,16}$

AL amyloidosis can manifest as diastolic dysfunction, conduction abnormalities or congestive cardiac failure. Our series show that incidence of CIDD is almost as frequent as AL amyloidosis in patients with plasma cell dyscrasias (31 vs $39 \%$ ). There appears to be no specific lightor heavy-chain predilection. Concurrent involvement by amyloid in other organs suggests the role of local tissue and cellular interactions in the morphologic manifestations of monoclonal Ig deposition. Not all patients with CIDD are clinically symptomatic.

The clinical manifestations include left ventricular hypertrophy (in the absence of hypertension), diastolic dysfunction and arrhythmias. Immunofluorescence and EM studies are diagnostic and should be considered in all patients with plasma cell dyscrasias undergoing myocardial biopsy and work-up.

\section{Conclusion}

CIDD is an uncommon complication of plasma cell dyscrasias. This diagnosis has to be considered in the differential diagnosis of such patients with cardiac dysfunction. Adequate sampling for EM and immunofluorescence studies of the myocardial biopsy is necessary to diagnose this entity. Clinically, these patients may be asymptomatic, at risk for developing arrhythmias or diastolic dysfunction. Our series also demonstrates the concurrent involvement of amyloid and light chain or light- and heavy-chain deposition in various organs sampled at the same time point. This may support the evolving theory of the role of local microenvironment in the pathogenesis of systemic diseases. 


\section{References}

1 Buxbaum JN, Chuba JV, Hellman GC, et al. Monoclonal immunoglobulin deposition disease: light chain and light and heavy chain deposition diseases and their relation to light chain amyloidosis. Clinical features, immunopathology and molecular analysis. Ann Intern Med 1990;112:455-464.

2 Buxbaum JN. Mechanisms of disease: monoclonal immunoglobulin deposition. Amyloidosis, immunoglobulin deposition disease, and light and heavy chain deposition disease. Hematol Oncol Clin N Am 1992; 6:323-346.

3 Buxbaum JN, Genega EM, Lazowski P, et al. Infiltrative nonamyloidotic monoclonal immunoglobulin light chain cardiomyopathy: an underappreciated manifestation of plasma cell dyscrasias. Cardiology 2000;93: 220-228.

4 Christou L, Hatzimichael EC, Sotsiou-Candila F, et al. A patient with multiple myeloma, amyloidosis and light-chain deposition disease in kidneys with a long survival. Acta Haematol 1999;101:202-205.

5 Daicker BC, Mihatsch MJ, Strom EH, et al. Ocular pathology in immunoglobulin deposition disease. Eur J Ophthalmol 1995;5:75-81.

6 Girelli CM, Lodi G, Rocca F. Kappa immunoglobulin deposition disease of the liver. Eur J Gastroenterol Hepatol 1998;10:429-430, 81.

7 Schattner A, Epstein M, Berrebi A, et al. Case report: multiple myeloma presenting as a diastolic heart failure with no evidence of amyloidosis. Am J Med Sci 1995;310:256-257.
8 Jego P, Paillard F, Ramee MP, et al. Congestive heart failure: revealing immunoglobulin deposition disease. Eur J Intern Med 2000;11:101-103.

9 Garton MJ, Walton S, Ewen SW. Systemic lambda lightchain deposition presenting with predominant cardiac involvement. Postgrad Med J 1993;69:588-591.

10 Grassi MP, Clerici F, Perin C, et al. Immunoglobulin deposition disease neuropathy resembling amyloid neuropathy in a multiple myeloma patient. Ital J Neurol Sci 1998;19:229-233.

11 Hamidou MA, Gires C, Moreau A, et al. Lambda Immunoglobulin deposition disease presenting as Sicca syndrome. Arthritis Rheum 1997;40:587-588.

12 Kasahara N, Tamura H, Matsumura O, et al. An autopsy case of immunoglobulin deposition disease. Intern Med 1994;33:216-221.

13 Gallo G, Goni F, Boctor F, et al. Light chain cardiomyopathy. Structural analysis of the light chain tissue deposits. Am J Pathol 1996;148:1397-1406.

14 Vidal R, Goni F, Stevens F, et al. Somatic mutations of the L12a gene in V-kappa(1) Immunoglobulin deposition disease: potential effects on aberrant protein conformation and deposition. Am J Pathol 1999;155: 2009-2017.

15 Keeling J, Teng J, Herrera GA. AL-amyloidosis and lightchain deposition disease light chains induce divergent phenotypic transformations of human mesangial cells. Lab Invest 2004;84:1322-1338.

16 Russell WJ, Cardelli J, Harris E, et al. Monoclonal light chain-mesangial cell interactions: early signaling events and subsequent pathologic effects. Lab Invest 2001;81:689-703. 